Provided for non-commercial research and education use. Not for reproduction, distribution or commercial use.

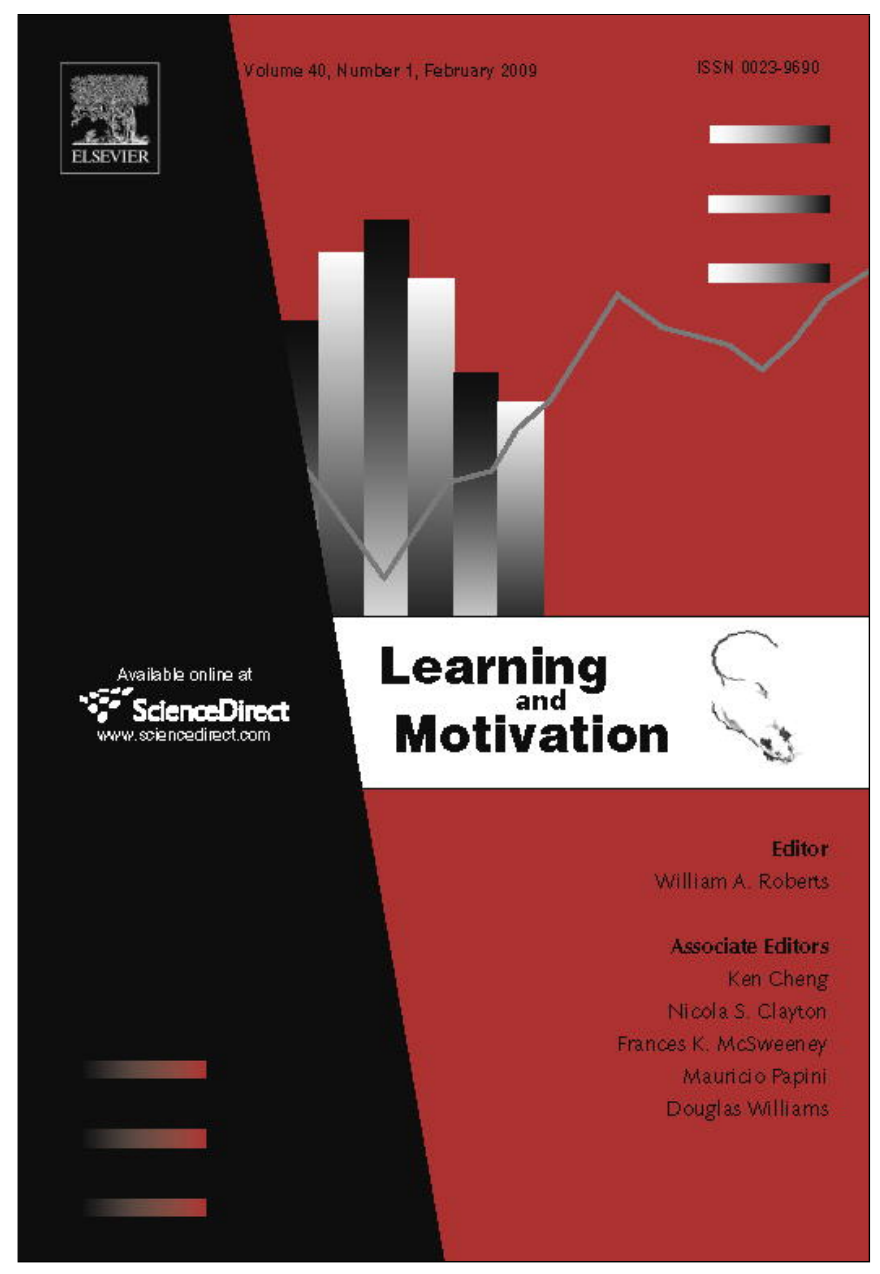

This article appeared in a journal published by Elsevier. The attached copy is furnished to the author for internal non-commercial research and education use, including for instruction at the authors institution and sharing with colleagues.

Other uses, including reproduction and distribution, or selling or licensing copies, or posting to personal, institutional or third party websites are prohibited.

In most cases authors are permitted to post their version of the article (e.g. in Word or Tex form) to their personal website or institutional repository. Authors requiring further information regarding Elsevier's archiving and manuscript policies are encouraged to visit:

http://www.elsevier.com/copyright 


\title{
Evidence of blocking with geometric cues in a virtual watermaze
}

\author{
Edward S. Redhead ${ }^{\mathrm{a}, *}$, Derek A. Hamilton ${ }^{\mathrm{b}}$ \\ ${ }^{a}$ School of Psychology, University of Southampton, Highfield, Southampton SO17 1BJ, UK \\ ${ }^{\mathrm{b}}$ University of New Mexico, Albuquerque, NM 87131, USA
}

\section{A R T I C L E I N F O}

\section{Article history:}

Received 4 October 2007

Revised 11 June 2008

Available online 9 August 2008

\section{Keywords:}

Virtual watermaze

Blocking procedure

Geometric cues

Non-geometric cues

\begin{abstract}
A B S T R A C T
Three computer based experiments, testing human participants in a non-immersive virtual watermaze task, used a blocking design to assess whether two sets of geometric cues would compete in a manner described by associative models of learning. In stage 1 , participants were required to discriminate between visually distinct platforms. In stage 2, additional spatial information was provided by the shape or the color of the walls of the pool. In a test trial, the platforms were removed and the spatial knowledge acquired regarding the position of the platform was assessed. Experimental groups were compared against control groups which did not receive stage 1 training. The unique color of the correct platform, in Experiments 1 and 3, disrupted learning about the colored walls but not the geometry of the pool. In Experiment 2, the correct platform was identifiable from its position within the three platform array. Learning the relative position of the correct platform within the array disrupted learning about its position relative to the geometry of the pool, but not to the colored walls. The results suggest that learning the position of a goal in relation to the geometry of the environment can be blocked but only by an alternative geometric cue.
\end{abstract}

(C) 2008 Elsevier Inc. All rights reserved.

Several experiments using both human (e.g., Chamizo, Aznar-Casanova, \& Artigas, 2003; Redhead \& Hamilton, 2007) and non-human participants (e.g., Redhead, Roberts, Good, \& Pearce, 1997; Roberts \& Pearce, 1999) have illustrated that the presence of a visible platform disrupts learning about the platform's position within a watermaze relative to the landmarks around the platform. Redhead

\footnotetext{
* Corresponding author. Fax: +44 (0) 2380594597.

E-mail address: er2@soton.ac.uk (E.S. Redhead).
} 
and Hamilton (2007) asked human participants to approach a visible platform in a computer generated virtual watermaze, and the position of the platform could also be encoded in relation to the cues around the watermaze. When the visible platform was removed, the participants spent no more time in the area of the pool previously occupied by the platform than would be expected by chance. Chamizo (2003) suggested that the failure to learn the spatial relationship between landmarks and platform position was a result of competition between cues. Such competition between spatial cues could be said to be similar to that seen within a typical conditioning experiment where one conditioned stimulus (CS) might be overshadowed or blocked by another CS. Chamizo (2003) suggested that the rules governing spatial learning were the same as those governing associative learning and that associative models such as the Rescorla and Wagner (1972) could be used to predict spatial learning.

Other experiments, however, have demonstrated that not all spatial learning follows the predictions of associative models. When the spatial cues were provided by the environment's shape (e.g., McGregor, Hayward, Pearce, \& Good, 2004; Pearce, Ward-Robinson, Aydin, Good, \& Fussell, 2001; Redhead \& Hamilton, 2007), spatial learning was not disrupted by the presence of a visible beacon. Redhead and Hamilton (2007) found that learning to approach a visible platform in a computer generated isosceles pool did not disrupt human participants' ability to learn the position of the platform in terms of the shape of the pool.

The lack of competition between cues predicted by associative models could be explained if geometric cues are impervious to disruption by other types of cues. Several authors (e.g., Cheng, 1986; Gallistel, 1990; Wang \& Spelke, 2002) have suggested that geometric cues are processed in a separate module. Indeed Doeller, King, and Burgess (2008) have shown in fMRI studies on participants using a virtual maze task that right posterior hippocampal activation was associated with learning boundary related locations, while right dorsal striatal activation reflected learning landmark related locations.

The main aim of the current paper is to assess whether opposing geometric cues compete in a manner predicted by associative models. Doeller and Burgess (2008) have shown that human participants in a virtual arena can learn the position of cues in relation to two separate elements of the same boundary without interaction. The current paper, however, will present two separate geometric cues, one formed by the shape of an array of identical objects within a virtual watermaze and the other by the shape of the watermaze itself.

The paper describes a series of experiments using a blocking design with human participants in a non-immersive virtual watermaze task. In stage 1 the participants were required to locate a platform using either a geometric or non-geometric cue. In stage 2, participants were provided with additional geometric or non-geometric cues. Finally, the additional cues are presented alone and the participants' ability to locate the platform is tested to investigate whether pre-exposure to the initial cues blocked learning about the additional cues presented in stage 2 .

\section{Experiment 1}

The aim of Experiment 1 was to test whether blocking was observed using human participants in a virtual water maze. Evidence for associative blocking among conspicuous distal landmarks has been reported in humans using a virtual Morris water task (Hamilton \& Sutherland, 1999). Redhead and Hamilton (2007) reported overshadowing of non-geometric cues by the presence of a visible platform, but not geometric cues. The current study will evaluate whether pre-exposure to the visible platform in a blocking procedure results in more disruption to learning geometric and non-geometric cues than mere compound exposure in an overshadowing procedure.

For the Experimental Blocking Groups the procedure consisted of two stages. In stage 1, over a series of learning trials, all participants were required to approach one of two visually discriminable platforms in the center of a circular pool and only one platform allowed escape from the pool. In stage 2 the participants were again asked to discriminate between the same two platforms but this time the platforms were placed in two corners of a triangular pool. For Group Landmark the pool was an equilateral triangle with one wall a different color from the other two. For Group Shape, the pool was an isosceles triangle with all of the walls the same color. Schematic layouts of the pools and platforms in stages 1 and 2 are illustrated in Fig. 1, and a participant view of the correct and incorrect platforms in 


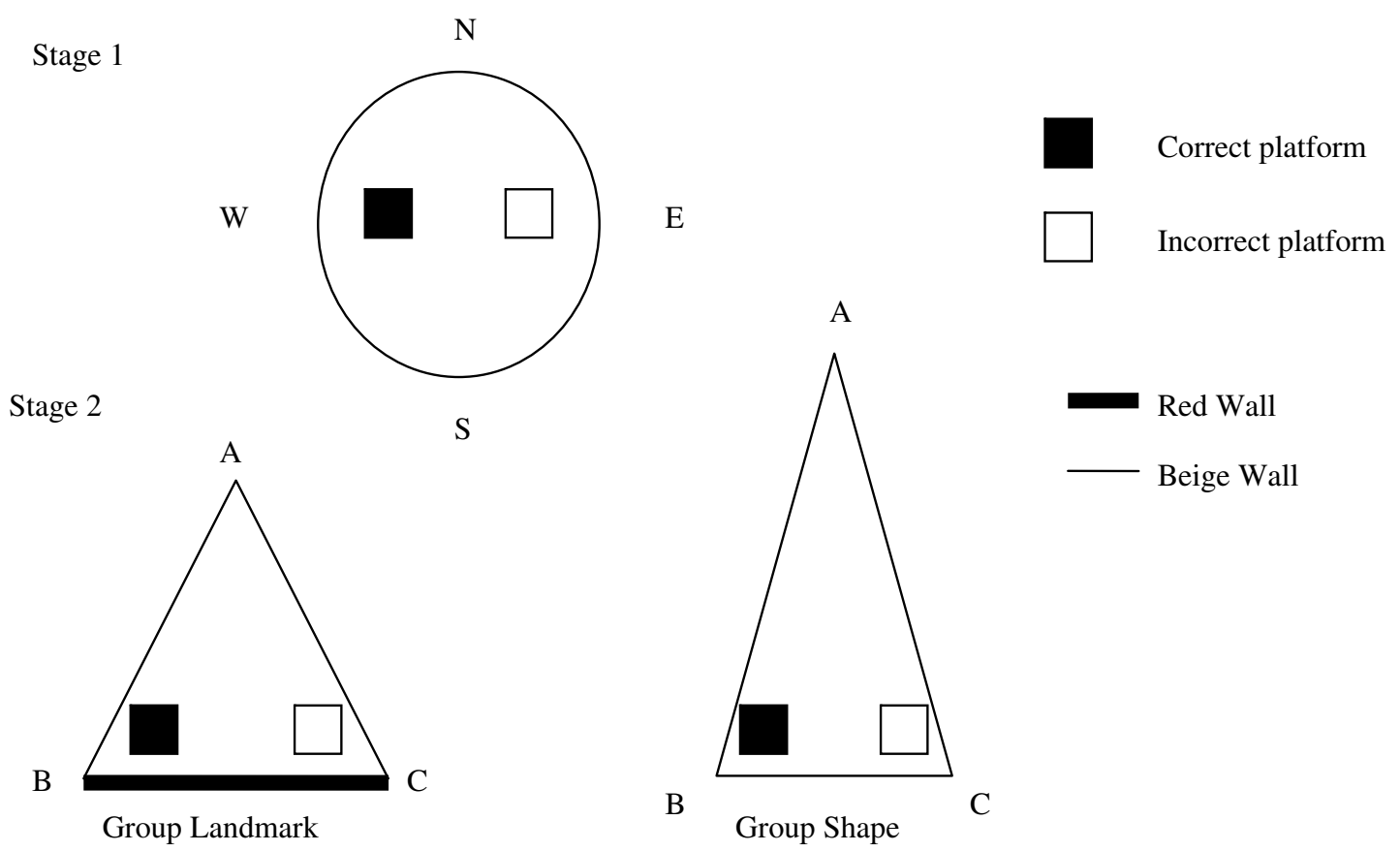

Fig. 1. Pool shape and platform position in stage 1 and in stage 2 for Group Landmark and Group Shape.
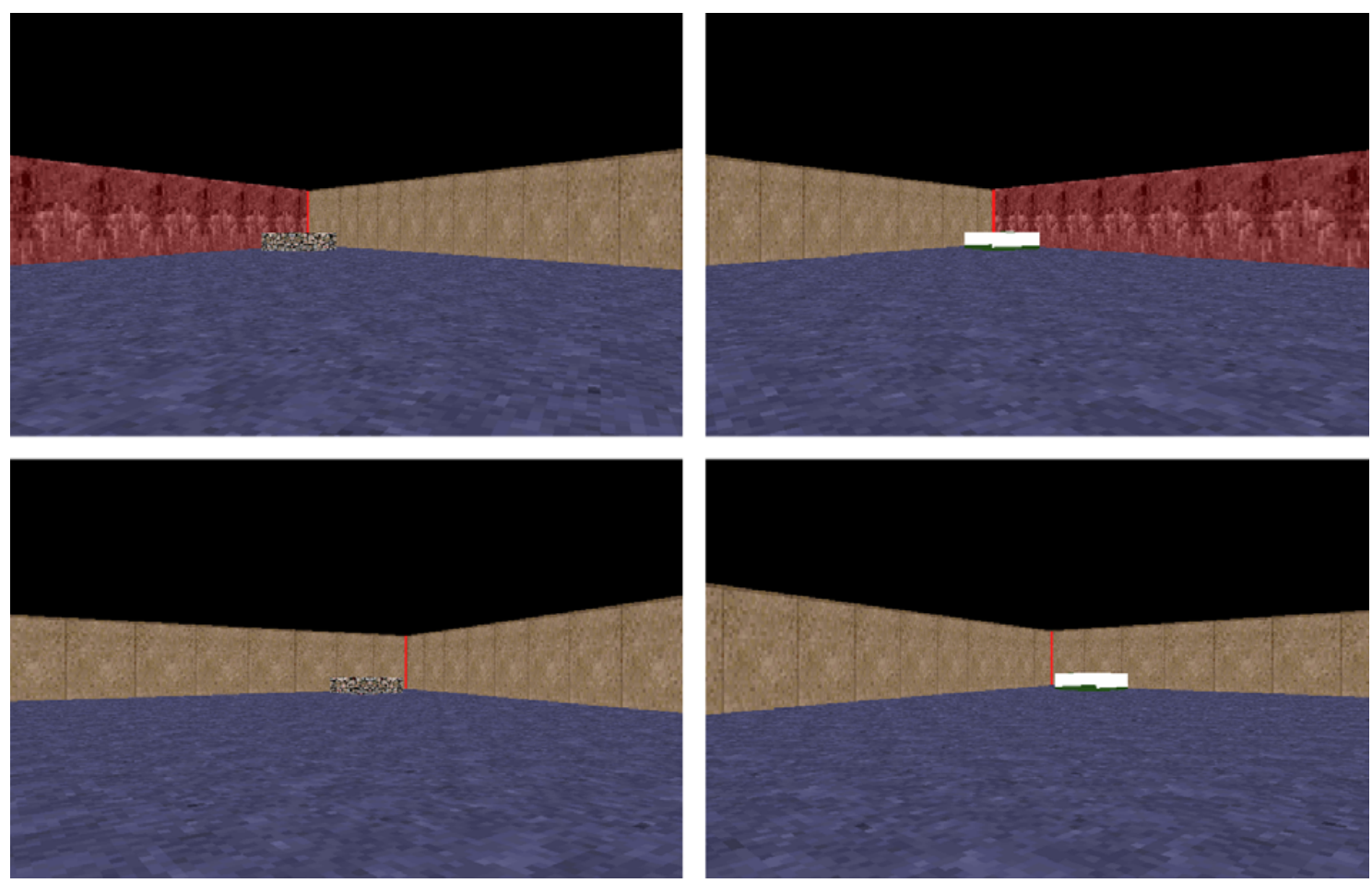

Fig. 2. Participant view in Group Landmark from center of pool of correct platform (upper left) and incorrect platform (upper right). Participant view in Group Shape from the same position of correct platform (lower left) and incorrect platform (lower right).

triangular pools of stage 2 can be seen in Fig. 2. Equilateral and Isosceles triangle pools were chosen as they offered both ambiguous and unambiguous geometric cues, respectively, while maintaining area and number of corners constant across the groups.

How well both blocking groups had learned the position of the platform in terms of the landmarks in the pool or the shape of the pool, respectively, was tested by removing the visible platforms and asking the participants to go to the area of the pool associated with the correct platform. The corre- 
sponding Overshadowing Control groups (Group Landmark Control and Group Shape Control) did not receive the stage 1 training. These groups where used to assess the effect of merely training the target cues in the presence of the visible platform.

\section{Methods}

\section{Participants}

There were 54 undergraduate students receiving $£ 1.50$ payment for participation with a mean age of 24.3 years, and age range between 18 and 32 years. Participants were divided into four groups, Group Landmark contained 4 males and 8 females, Group Shape contained 3 males and 9 females, Group Landmark Control contained 5 males and 10 females and Group Shape Control contained 6 males and 9 females.

\section{Materials}

The experiment was performed in a research cubicle (length $2.4 \mathrm{~m}$, width $1.3 \mathrm{~m}$, height $2 \mathrm{~m}$ ) containing a chair in front of a $1.3 \mathrm{~m}$ wide work bench attached to the wall opposite the entrance to the cubicle. A 15-inch color computer monitor and keyboard were placed on the work bench. The monitor was connected to an IBM compatible PC placed beneath the bench. The virtual environment used in stage 1 for Group Landmark and Group Shape consisted of a circular pool, 75 units in diameter, with a beige colored wall 15 units high. It took approximately $3 \mathrm{~s}$ to travel the diameter of the pool. The pool contained two platforms during acquisition training, a correct and an incorrect platform. The correct platform was black and grey and was placed 25 units from the center of the pool toward a point designated as West. The incorrect platform was white and was placed 25 units from the center toward a point designated as East. Touching the correct platform terminated the trial. The visible platforms were cubes, 5 units in height, width and length. An opaque, blue pattern was used to create the surface of the pool. Beyond the walls and above the pool the background was black, and no room contours were visible.

Auditory feedback consisted of a bell when the correct platform was reached, a discordant tone when the incorrect platform was touched, and the sound of moving water accompanied forward motion in the pool. Navigation was controlled using the keyboard arrow keys. The UP arrow key was used to control forward motion, the LEFT and RIGHT keys controlled rotation. Backward navigation was not possible. It took approximately $1.5 \mathrm{~s}$ to complete one complete rotation.

In stage 2, presented to all groups, the virtual environment consisted of a triangular pool. The triangle for Group Landmark and Group Landmark Control was equilateral, with each wall 100 units in length and 15 units in height. Two of the walls were beige in color and the other red. The center of the correct platform was located 17 units from corner $B$ on a line at an angle of $34^{\circ}$ from the wall between $\mathrm{B}$ and $\mathrm{C}$. The center of the incorrect platform was placed at a similar distance and angle from corner $\mathrm{C}$. For Group Shape and Group Shape Control, the pool was an isosceles triangle, with the long walls 140 units in length and the short wall 60 units. All walls were beige in color and 15 units in height. The center of each platform was placed at a distance of 7.5 units from the wall between corners $B$ and C. For Group Shape, the center of the correct platform was located 17 units from corner B on a line at an angle of $43^{\circ}$ from the wall between corner B and C. The center of the incorrect platform was placed at a similar distance and angle from corner $C$ and at a distance of 7.5 units from the wall between B and C. For all groups, the south edge of both platforms ran parallel to the wall between B and $\mathrm{C}$.

\section{Procedure}

Participants were led into the cubicle and asked to sit in front of the computer after which the experimenter left the room. The following instructions were given via the computer screen to participants of Group Landmark and Group Shape:

In this experiment you will view a computer-generated environment on the monitor. You will be viewing the environment from a first-person perspective and you can move through the environment using the arrow keys on the keyboard (UP, LEFT, AND RIGHT). You will be placed in a circular 
pool of water from which you must escape by climbing onto a platform. When you touch the platform you will be stopped, raised out of the water, and you will see a message saying that you have found the platform. There will be 2 platforms in the pool, only one platform allows escape from the pool. Your goal is to locate that platform and climb onto it as quickly as possible. You will be on the platform for a few moments during which time you can scan around the pool. The screen will then fade out and you will begin another trial. You will complete several trials. On each trial you will begin facing the wall of the pool. Because you cannot move backwards you will need to turn before you can move out into the pool.

Press the space bar when you are ready to start.

Once participants pressed the space bar, the computer screen displayed the wall of the watermaze at one of four release points designated as North East, North West, South West or South East in the pool. Over the 10 acquisition trials, each release point was used at least twice. On reaching the correct platform, a bell sounded and the words "You have gained 10 points" appeared on the screen. The 10 points were added to a total displayed in the top right corner of the screen. Participants were then placed on top of the platform for $5 \mathrm{~s}$ before the screen went dark for $1 \mathrm{~s}$, and participants were again placed facing a corner for the start of the next acquisition trial. If participants touched the incorrect platform, a discordant tone was sounded, the message "You have lost 10 points" was displayed on the screen and 10 points were deducted from the total. The participants were allowed to continue each trial until they had reached the correct platform. The time to touch the correct platform was recorded for each acquisition trial. There were 10 trials after which the program ended and the participants were required to alert the experimenter outside. The experimenter would then start the program for stage 2 .

Group Landmark Control and Group Shape Control did not receive stage 1 training and when entering the cubicle proceeded straight to stage 2. Prior to beginning the navigation task all, participants received a similar set of instruction to those shown above except the pool was described as being either an isosceles or equilateral triangle.

In stage 2, participants were either placed in an equilateral triangle with one red wall and two beige walls (Group Landmark and Group Landmark Control) or an isosceles triangle (Group Shape and Group Shape Control) and were instructed to approach and touch the "correct platform". All groups received nine acquisition trials followed by a test trial where the platforms had been removed. At the start of the test trial, participants began in the center of the pool, facing corner A. They were not given any new instructions. There were no platforms in the pool, and the trial lasted for $45 \mathrm{~s}$. Latency to cross and time spent in two areas of the pool (length 10 units, width 10 units) centered on the positions of the platforms were recorded during the test trials.

\section{Results and discussion}

All statistical tests were evaluated with respect to an alpha value of 0.05 . The Groups Landmark and Shape were analysed separately with their respective control groups to assess the effects of pre-exposing the participants to a visible platform.

\section{Group Landmark and Landmark Control}

The mean escape latencies for Group Landmark decreased over the course of stage 1 training (Group Landmark Trial $1 M=8.89, S D=5.62$, and Trial $10 M=3.76, S D=2.73$ ). A one-way repeated measures analysis of variance (ANOVA) was performed on the escape latencies of Group Landmark, with trial the independent variable. There was a significant effect of trial, $F(9,99)=11.10$.

The mean escape latencies in stage 2 stayed fairly constant for Group Landmark (Trial $1 M=10.09$, $S D=3.43$, and Trial $9 M=7.01, S D=4.11$ ) but decreased for Group Landmark Control (Trial 1 $M=17.80, S D=6.82$, and Trial $9 M=3.90, S D=4.23$ ). The group scores were analysed using a twoway mixed design ANOVA with group (between) and trial (within) the independent variables. There was no main effect of group, $F<1$, a significant effect of trial, $F(8,200)=8.98$, and a significant interaction between group and trial, $F(8,200)=3.65$. Further analysis of the simple main effects (Keppel, 1973 ) revealed that there was an effect of group only on trial $1, F(1,225)=10.06$, and that there 
was an effect of trial only for Group Landmark Control, $F(8,200)=12.00$. The longer initial latencies of participants in Group Landmark Control could be explained by their lack of training in stage 1. However, by the end of stage 2, their latencies were similar to Group Landmark.

The group means for the time spent in the correct and incorrect platform areas, represented as a percentage of the total time during the test trials, are shown in the left hand panel of Fig. 3. There appears little difference in the percentage time spent in the correct platform compared to the incorrect platform for either Group Landmark or Group Landmark Control. A two-way mixed design ANOVA with percentage time as the dependent variable and group (between) and platform type (within) as the independent variables confirmed this impression. There was no overall effect of group, $F(1,25)=3.11$, or platform type, $F<1$, and the interaction between group and platform type was not significant, $F<1$.

The group mean for the latency to cross the correct and incorrect platforms during the test trial are shown in the right hand panel of Fig. 3. The latency to cross the correct platform was slightly longer than latency to cross the incorrect platform for Group Landmark, but, for Group Landmark Control, latency to cross the correct platform was much shorter than latency to cross the incorrect platform. A two-way mixed design ANOVA with latency to cross the platform as the dependent variable and group (between) and platform type (within) as the independent variables confirmed these impressions. There was an overall effect of group, $F(1,25)=16.20$, no effect of platform type, $F(1,25)=3.64$, and the interaction between Group and Platform Type was significant, $F(1,25)=8.04$. Further analysis of the simple main effects revealed that there was an effect of platform type for Group Landmark Control, $F(1,25)=11.25$, but not for Group Landmark, $F<1$. There was no effect of group for the correct platform, $F<1$, but there was an effect for the incorrect platform, $F(1,50)=19.94$.

\section{Group Shape and Shape Control}

The mean escape latencies for Group Shape decreased over the course of stage 1 training (Trial 1 $M=9.54, S D=4.62$, and Trial $10 M=3.24, S D=1.82$ ). A one way repeated measures ANOVA was performed on the escape latencies with trial the independent variable. There was a significant effect of trial, $F(9,99)=7.06$.

The mean escape latencies in stage 2 decreased over the trials for both Group Shape (Trial $1 M=9.0$, $S D=5.66$, and Trial $9 M=5.88, S D=3.06$ ) and Group Shape Control (Trial $1 M=14.72, S D=4.02$, and Trial $9 M=5.21, S D=1.83$ ). The groups were compared using a two-way mixed design ANOVA with group (between) and trial (within) the independent variables. There was no main effect of group,

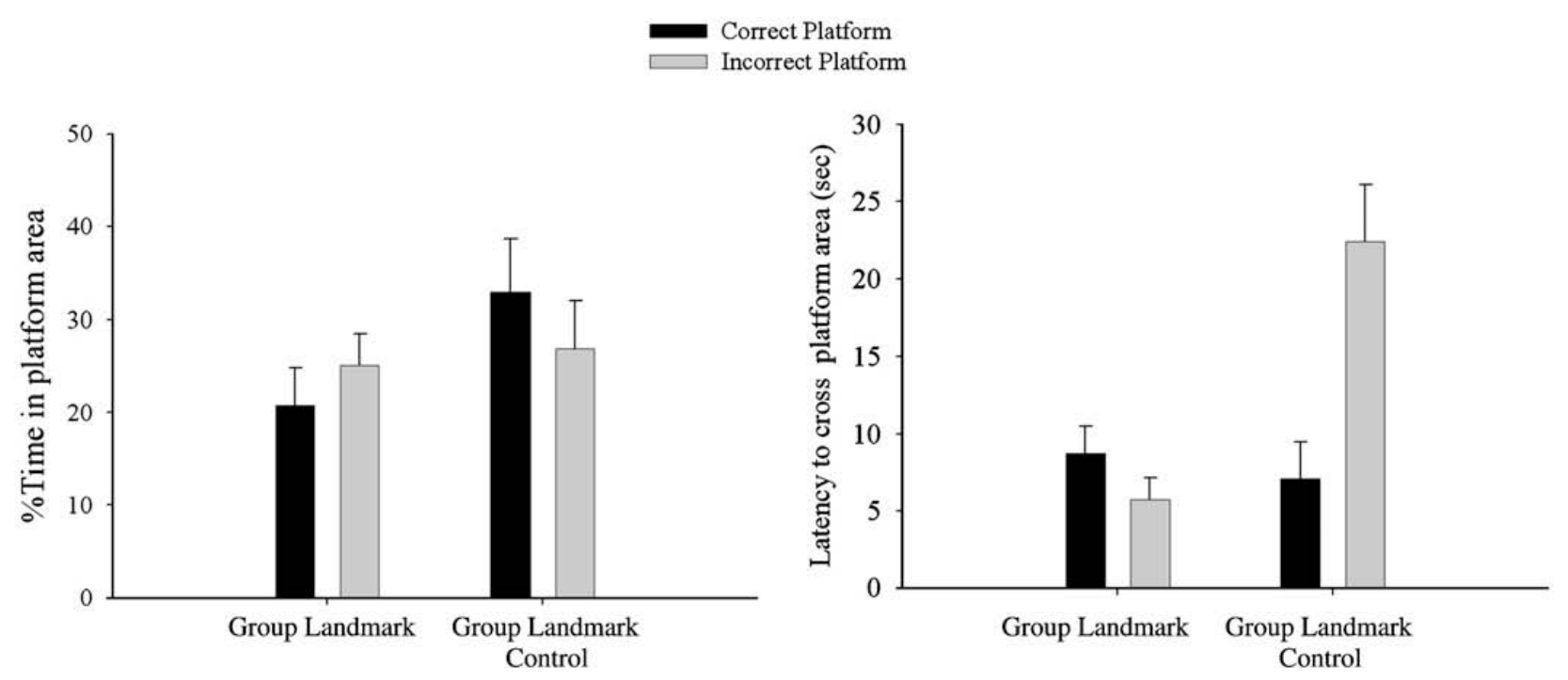

Fig. 3. Mean percentage time spent in correct (black bars) and incorrect (grey bars) platforms for Group Landmark and Group Landmark Control during test trial (left hand panel). Mean latency to cross correct (black bars) and incorrect (grey bars) platforms for Group Landmark and Group Landmark Control during test trial (right hand panel). The standard error bars are the standard error of the mean. 
$F<1$, a significant effect of trial, $F(8,200)=17.16$, and a significant interaction between group and trial, $F(8,200)=5.59$. Further analysis of the simple main effects revealed that there was an effect of group only on Trial $1, F(1,225)=26.97$, and that there was an effect of trial for both Group Shape Control, $F(8,200)=20.70$, and Group Shape, $F(8,200)=2.05$. The pattern of results for Group Shape Control was similar to that seen in Group Landmark Control and can also be explained by the participants having no training previous to stage 2 . The important factor is that by the end of stage 2 there was no difference between the respective control and experimental groups.

The group means for the time spent in the correct and incorrect platforms, represented as a percentage of the total time during the test trials, are shown in the left hand panel of Fig. 4 . The percentage time spent was higher in the correct platform compared to the incorrect platform for both Group Shape and Group Shape Control. A two-way mixed design ANOVA with percentage time as the dependent variable and group (between) and platform type (within) as the independent variables confirmed this appearance. There was no overall effect of group, $F(1,25)=1.35$, an effect of platform type, $F(1,25)=12.30$, and no interaction between group and platform type, $F<1$.

The group mean for the latency to cross the correct and incorrect platforms during the test trial are shown in the right hand panel of Fig. 4. The latency to cross the correct platform was shorter than the latency to cross the incorrect platform for both Group Shape and Group Shape Control. A two-way mixed design ANOVA with latency to cross the platform as the dependent variable and group (between) and platform type (within) as the independent variables confirmed this impression. There was no overall effect of group, $F(1,25)=3.53$, an effect of platform type, $F(1,25)=7.07$, and a non-significant interaction between Group and Platform Type, $F<1$.

The results of the test stage confirm previous findings that within a blocking procedure, pre-exposure to a visible platform does not disrupt learning about the position of a goal in relation to the shape of the pool (e.g., Hayward, McGregor, Good, \& Pearce, 2003) but it does in relation to the landmarks around the pool (e.g., Roberts \& Pearce, 1999). The results of Group Landmark Control suggest that the presence of the visible platform in stage 2 alone is enough to disrupt learning about the position of the platform in relation to the landmark, as there was no difference in the time spent in the correct and incorrect platform areas. This finding replicates those of the overshadowing experiments performed by Redhead and Hamilton (2007). However, latency to cross the platform area, not measured in the Redhead and Hamilton study, indicated that Group Landmark Control crossed the correct platform area before the incorrect platform area, whereas this was not true for Group Landmark. There is, therefore, at least some evidence that pre-exposure to the visible platform as part of a blocking design

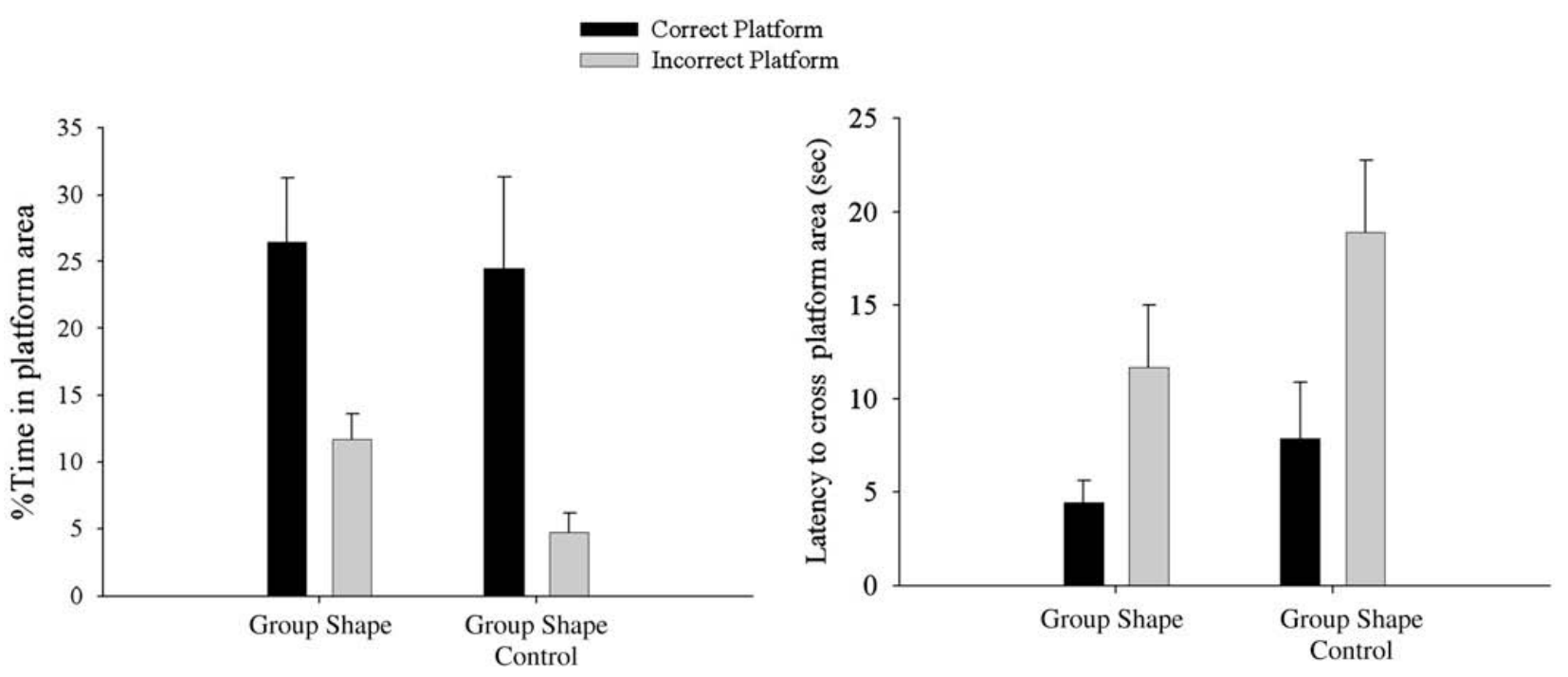

Fig. 4. Mean percentage time spent in correct (black bars) and incorrect (grey bars) platforms for Group Shape and Group Shape Control during test trial (left hand panel). Mean latency to cross correct (black bars) and incorrect (grey bars) platforms for Group Shape and Group Shape Control during test trial (right hand panel). The standard error bars are the standard error of the mean. 
is more effective at disrupting learning about the landmark than it is as part of a compound in an overshadowing design.

The fact that pre-exposure to a visible platform does not block learning about shape supports Cheng's (1986) suggestion that there is a separate module which processes shape. Such a finding does not rule out the possibility that associative mechanisms govern learning about shape in relation to platform position, but merely indicates that landmarks and shape do not compete for associative strength. In order to test whether two sets of geometric cues compete with each other, in Experiment 2 participants were pre-exposed to an array of three identical platforms placed into the shape of a notional isosceles triangle. Experiment 2 tested whether learning the shape of the array would block learning about the position of the platform with relation to the shape of the pool.

\section{Experiment 2}

Experiment 2 consisted of four groups, Group Landmark Shape and Group Shape Shape and their respective control groups. Only the Experimental Groups received stage 1 training. All participants in the experimental groups were placed in a circular pool containing three identical platforms and were required to approach the correct platform. The platforms were placed at the corners of a notional isosceles triangle, and the correct platform was placed at the intersection of the two long sides of the triangle. Schematic layouts of the pools and platforms are illustrated in Fig. 5. Both animals and humans have been shown to be able to use triangular object arrays to locate goals (e.g., Benhamou \& Poucet, 1998; Spetch, Cheng, \& MacDonald, 1996).

There has been a recent debate over whether local or global cues are encoded when learning geometric information (e.g., Cheng, 2005; Pearce, Graham, Good, Jones, \& McGregor, 2006) and whether the same class of cues are used to encode enclosures and arrays (Spetch et al., 1996; Wang \& Spelke, 2000). For example, the correct platform in the array might be identified in terms of the local cues such as the platform with the farthest distance between itself and the other two. In terms of global cues, it might be described as being at the apex of the isosceles triangle. Although it is beyond the scope of the studies here to define which type of cue participants used, the absence or presence of blocking may provide evidence as to whether the same type of cue is used to encode geometric information from an enclosure and an array.

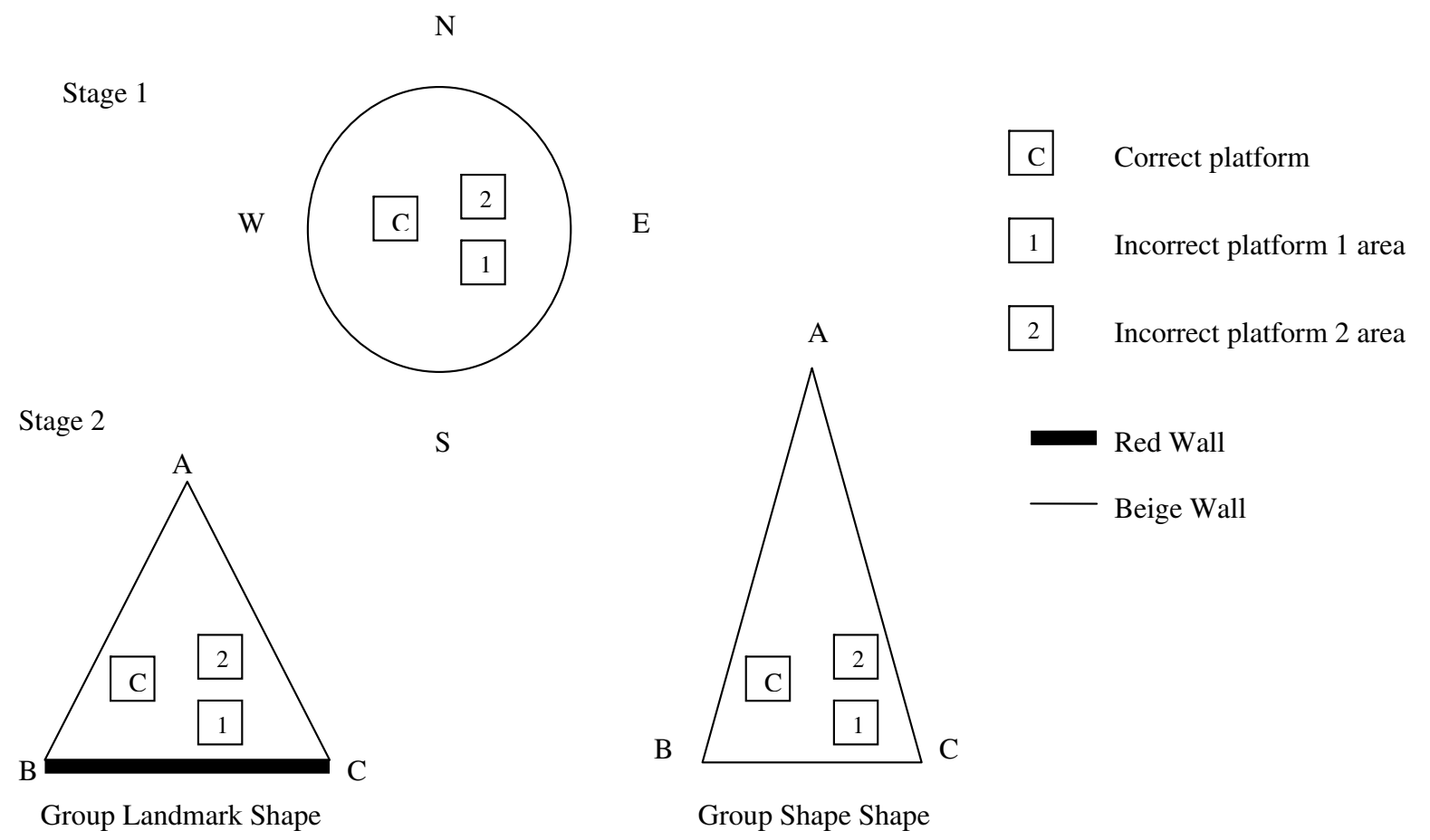

Fig. 5. Pool shape and platform position in stage 1 and in stage 2 for Group Landmark Shape and Group Shape Shape. 
All participants received training in stage 2. For Group Landmark Shape and Group Landmark Shape Control the three platforms were placed into an equilateral triangular pool with one of the walls red and the others beige. Participants could either continue to locate the correct platform in terms of its position within the array of platforms or in relation to the red wall of the pool. For Group Shape Shape and Group Shape Shape Control the three platforms were placed into an isosceles shaped pool. Participants could either locate the correct platform in relation to its position within the array or locate its position in relation to the shape of the pool. In the test stage, the platforms were removed and the participants were requested to go to the area of the pool in which the correct platform had been placed. If the shape of an array competes for associative strength with the shape of the environment in the manner described by associative models (Rescorla \& Wagner, 1972) then Group Shape Shape should spend no more time in the correct platform of the pool than in the incorrect platforms.

\section{Method}

\section{Participants}

Participants were 54 undergraduate students, receiving payment of $£ 1.50$ for participation, divided into 4 groups: Group Landmark Shape, Group Landmark Shape Control, Group Shape Shape and Group Shape Shape Control. Within each experimental group, there were 2 males and 10 females; within Group Landmark Shape Control, there were 4 males and 11 females, and, within Group Shape Shape Control, there were 5 males and 10 females. The mean age was 21.8 years (range 18-24 years). Participants were not permitted to take part in Experiment 2 if they had previously completed Experiment 1.

\section{Materials and apparatus}

Materials and apparatus details were the same as in Experiment 1 with the exception that there were three platforms arranged at the points of a notional isosceles triangle. In stage 1 the axis of the triangle ran through the center of the circular pool between points designated as East and West. The platforms were all black and grey and their centers lay 15 units from the center of the pool. The correct platform lay to the west of the center of the pool and the incorrect platforms lay to the east of the center of the pool. The distance between the centers of incorrect platform 1 and incorrect platform 2 was 15 units. The distance between the center of the correct platform and both of the incorrect platforms was 30 units. In stage 2, the same spatial arrangement of platforms was placed in a triangular pool. For Group Landmark Shape and Group Landmark Shape Control, the pool consisted of the equilateral triangle presented to the participants of Group Landmark in Experiment 1. The center of the correct platform was located 37 units from corner B on a line at an angle of $28^{\circ}$ from the wall between corner $\mathrm{B}$ and $\mathrm{C}$ and at a distance of 18 units on a line perpendicular to this wall. The center of incorrect platform 1 was placed at a distance of 38 units from corner $C$ at an angle of $15^{\circ}$ from the wall between $B$ and $C$ and at a distance of 10 units from this wall. Finally the center of incorrect platform 2 was placed at a distance of 44 units from corner $\mathrm{C}$ at an angle of $34^{\circ}$ from the wall between B and C and 25 units on a line perpendicular to the wall. For Groups Shape Shape and Shape Shape Control the pool consisted of the isosceles triangle presented to the participants of Group Shape in Experiment 1. The center of the correct platform was located 23 units from corner $\mathrm{B}$ on a line at an angle of $50^{\circ}$ from the wall between corner $\mathrm{B}$ and $\mathrm{C}$ and at a distance of 18 units on a line perpendicular to this wall. The center of incorrect platform 1 was placed at a distance of 20 units from corner $C$ at an angle of $28^{\circ}$ from the wall between $B$ and $C$ and at a distance of 10 units on a line perpendicular to this wall. Finally, the center of incorrect platform 2 was placed at a distance of 30 units from corner $C$ at an angle of $55^{\circ}$ from the wall between $B$ and $C$ and 25 units on a line perpendicular to the wall. The south edge of each platform ran parallel to the wall between corners $\mathrm{B}$ and $\mathrm{C}$.

\section{Procedure}

Participants were instructed to locate the platform which allowed escape from the pool. There were 12 acquisition trials in both stages 1 and 2 . All other procedural details were the same as Experiment 1. 
Results and discussion

The Groups Landmark Shape and Shape Shape were analysed separately with their respective control groups to assess the effects of pre-exposing the participants to a visible platform.

\section{Group Landmark Shape and Landmark Shape Control}

The mean escape latencies for Group Landmark Shape decreased over the course of stage 1 training (Trial $1 M=14.60, S D=11.31$, and Trial $12 M=4.27, S D=1.55$ ). A one-way repeated measures analysis of variance (ANOVA) was performed on the escape latencies of Group Landmark Shape, with trial the independent variable. There was a significant effect of trial, $F(11,121)=7.52$.

The mean escape latencies in stage 2 decreased for both Group Landmark Shape (Trial $1 M=16.84$, $S D=13.47$, and Trial $12 M=8.26, S D=2.50$ ) and Group Landmark Shape Control (Trial $1 M=15.84$, $S D=13.64$, and Trial $12 M=5.82, S D=3.47$ ). The group scores were analysed using a two-way mixed design ANOVA with group (between) and trial (within) the independent variables. There was no main effect of group, $F<1$, a significant effect of trial, $F(11,275)=4.47$, and a non-significant interaction between group and trial, $F(11,275)=1.30$.

The group means for the time spent in the correct and incorrect platform areas, represented as a percentage of the total time during the test trials, are shown in the left hand panel of Fig. 6 . The percentage time spent was higher in the correct platform compared to the incorrect platforms for both Group Landmark Shape and Group Landmark Shape Control. A two-way mixed design ANOVA with percentage time as the dependent variable and group (between) and platform type (within) as the independent variables confirmed this impression. There was no main effect of group, $F(1,25)=3.76$, there was an effect of platform type, $F(2,50)=13.04$, and the interaction between group and platform type was not significant, $F<1$. Newman-Keuls tests on the time spent in the platform areas by both groups combined confirmed that participants spent more time in the correct platform than in both incorrect platform $1, q^{n k}(50)=5.62$, and incorrect platform $2, q^{n k}(50)=6.56$.

The group mean for the latency to cross the correct and incorrect platforms during the test trial are shown in the right hand panel of Fig. 6 . The latency to cross the correct platform was shorter than the latency to cross the incorrect platforms for both Group Landmark Shape and Group Landmark Shape Control. A two-way mixed design ANOVA with latency to cross the platform as the dependent variable and group (between) and platform type (within) as the independent variables confirmed this impression. There was a main effect of group, $F(1,25)=11.01$, an effect of platform type, $F(1,25)=5.95$, but the interaction between Group and Platform Type was not significant, $F<1$.
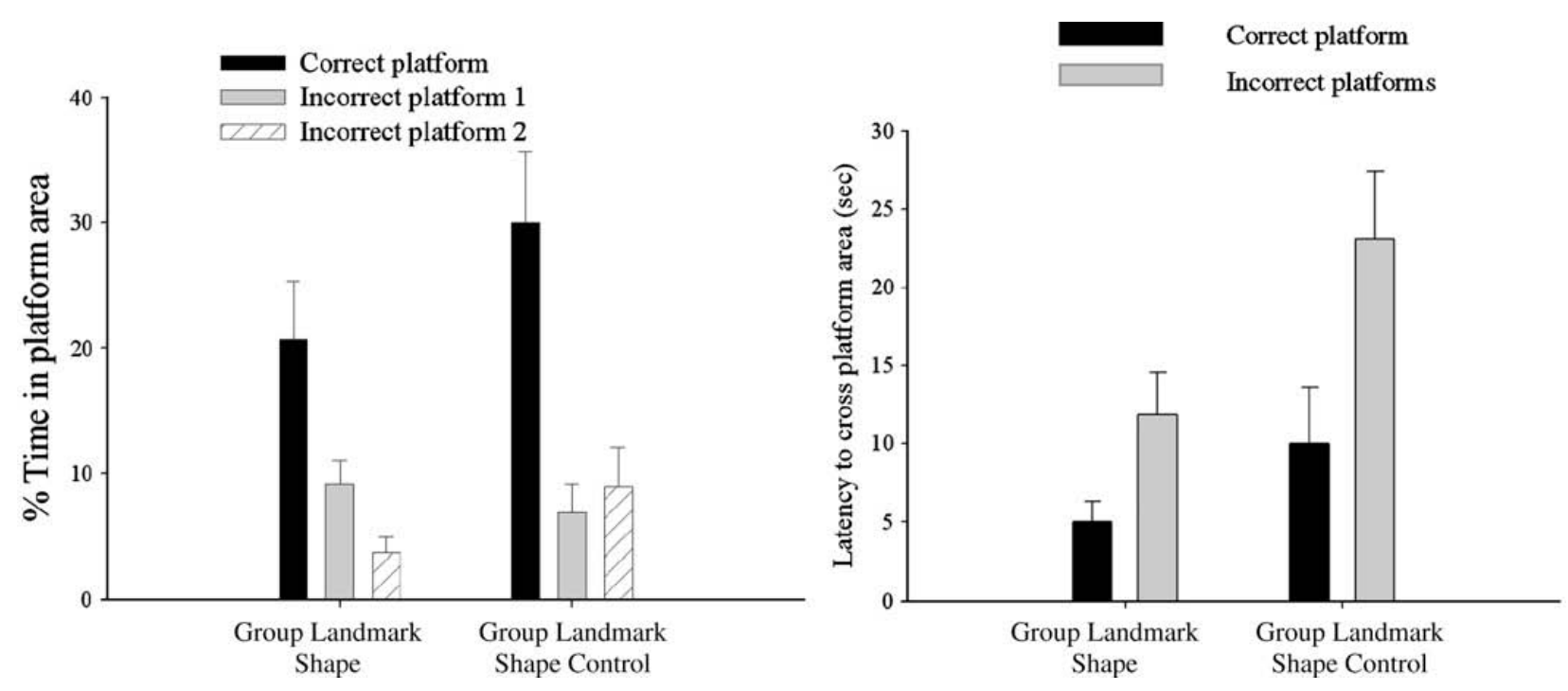

Fig. 6. Mean percentage time spent in correct (black bars) and incorrect (grey bars) platforms for Group Landmark Shape and Group Landmark Shape Control during test trial (left hand panel). Mean latency to cross correct (black bars) and incorrect (grey bars) platforms for Group Landmark Shape and Group Landmark Shape Control during test trial (right hand panel). The standard error bars are the standard error of the mean. 


\section{Group Shape Shape and Shape Shape Control}

The mean escape latencies for Group Shape Shape decreased over the course of stage 1 training (Trial $1 M=15.26, S D=12.11$, and Trial $12 M=5.36, S D=2.07$ ). A one way repeated measures ANOVA was performed on the escape latencies with trial the independent variable. There was a significant effect of trial, $F(11,121)=2.60$.

The mean escape latencies in stage 2 decreased over the trials for both Group Shape Shape (Trial 1 $M=16.28, S D=9.46$, and Trial $12 M=10.18, S D=7.49$ ) and Group Shape Shape Control (Trial 1 $M=17.38, S D=6.20$, and Trial $12 M=8.92, S D=9.86$ ). The groups were compared using a two-way mixed design ANOVA with group (between) and trial (within) the independent variables. There was no main effect of group, $F<1$, a significant effect of trial, $F(11,275)=7.52$, but no significant interaction between group and trial, $F(11,275)=1.65$.

The group means for the time spent in the correct and incorrect platforms, represented as a percentage of the total time during the test trials, are shown in the left hand panel of Fig. 7. There was no difference in the percentage time spent in the correct platform compared to the incorrect platforms for Group Shape Shape, but participants in Group Shape Shape Control did spend more time in the correct platform area. A two-way mixed design ANOVA with percentage time as the dependent variable and group (between) and platform type (within) as the independent variables confirmed this appearance. There was an effect of group, $F(1,25)=6.24$, an effect of platform type, $F(2,50)=4.60$, and a significant interaction between group and platform type, $F(2,50)=6.67$. Further analysis of the simple main effects revealed that there was an effect of platform type for Group Shape Shape Control, $F(2,50)=9.87$, but not for Group Shape Shape, $F(2,50)=1.40$. Newman-Keuls tests confirmed that participants in Group Shape Shape Control spent more time in the correct platform than in both incorrect platform $1, q^{n k}(50)=5.24$, and incorrect platform $2, q^{n k}(50)=6.18$. There was a significant effect of group for the correct platform, $F(1,75)=19.10$, but none for incorrect platforms 2 or $3, F s<1$.

The group mean for the latency to cross the correct and incorrect platforms during the test trial are shown in the right hand panel of Fig. 7. The latency to cross the correct platform was shorter than the latency to cross the incorrect platform for Group Shape Control but not for Group Shape Shape. A twoway mixed design ANOVA with latency to cross the platform as the dependent variable and group (between) and platform type (within) as the independent variables confirmed this impression. There was no main effect of group or platform type, $F<1$, but the interaction between Group and Platform Type was significant, $F(1,25)=6.34$. Further analysis of the simple main effects revealed that there was an effect of platform type for Group Shape Shape Control, $F(1,25)=5.93$, but not for Group Shape Shape, $F(1,25)=1.26$. There was a significant effect of group for latency to cross the incorrect platforms, $F(1,50)=5.93$, but not the correct platform, $F(1,50)=1.90$.
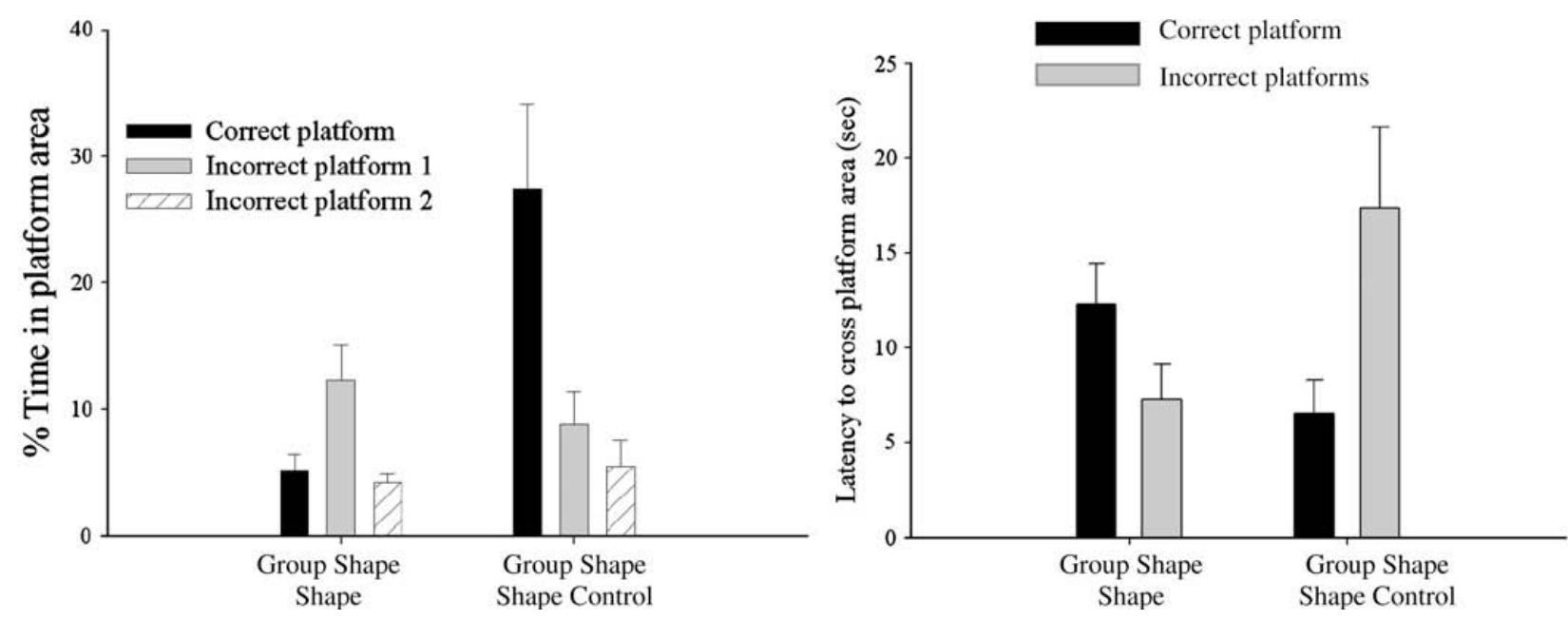

Fig. 7. Mean percentage time spent in correct (black bars) and incorrect (grey bars) platforms for Group Shape Shape and Group Shape Shape Control during test trial (left hand panel). Mean latency to cross correct (black bars) and incorrect (grey bars) platforms for Group Shape Shape and Group Shape Shape Control during test trial (right hand panel). The standard error bars are the standard error of the mean. 
The test results for Group Shape Shape and Group Shape Shape Control suggest that pre-exposure to the position of the correct platform in relation to the shape of the array of platforms, in stage 1 , blocked learning about the platform's position in relation to the shape of the pool in stage 2. Exposure to the shape of the platform array in stage 2 alone, experienced by Group Shape Shape Control in an overshadowing procedure, did not disrupt learning about the shape of the pool. Thus, when competing spatial cues, within a blocking design, are both geometric, learning is predicted by the rules of associative models (e.g., Rescorla \& Wagner, 1972).

The other important finding from Experiment 2 was that exposure to the shape array neither blocked nor overshadowed learning about the position of the platform in terms of the red wall. These findings suggest that learning the position of a goal in relation to non-geometric cues is impervious to disruption by geometric cues. The results also confirm those of Hayward, Good, and Pearce (2004), which showed that learning to find a hidden platform using the shape of the pool did not disrupt learning the hidden platform's position in relation to the landmarks around the edge of the pool.

There were two incidences of group differences during the test trials between the respective experimental and control groups. Overall, Group Landmark Shape Control's latencies to cross either platform were longer than those of Group Landmark Shape. It is not possible to say why this was the case, but, crucially, latency to cross the correct platform was shorter than that to cross the incorrect platforms for both groups. There was also a main effect of group in the analysis of time spent in the platform area between the Shape groups. Looking at the simple main effects, it is clear that Group Shape Shape Control spent more time in the correct platform area than Group Shape Shape, which fits with the main finding that pre-exposure the shape of the array blocked learning the shape of the arena.

It must be noted that to maintain the spatial relationships between the three platforms from stage 1 to stage 2, the position of the correct platform was located farther from the corners of the pool than in Experiment 1. This may have made it more difficult for Group Shape Shape to locate the position of the correct platform, particularly if the participants were using a strategy of searching close to the walls. However, the fact that the participants of the other three groups spent more time in the correct rather than the incorrect platform area suggests that the increased distance to the intersection of the walls did not make it impossible to locate the correct platform's position. A further difference between Group Shape in Experiment 1 and Group Shape Shape in Experiment 2 is that participants in the latter had to negotiate past two incorrect platforms instead of one. The paths taken to avoid losing points from contact with the two incorrect platforms could have resulted in restricted views of the pool, thus allowing Group Shape Shape less opportunity to learn the overall shape of the isosceles pool in comparison to Group Shape in Experiment 1. Experiment 3 was designed to determine whether the addition of a further platform or the location of the platforms in comparison to the corners hindered participants of Group Shape Shape. In Experiment 3, the number and position of the platforms were the same as in Experiment 2, but the platforms were not visually identical. Similar to Experiment 1, the correct platform was a different color from the incorrect platforms. Such information might be expected to block learning about the position of the platform in relation to the red wall but not in relation to the shape of pool, as was the case in Experiment 1.

\section{Experiment 3}

There were four groups in Experiment 3, Group Landmark Beacon and Group Shape Beacon and their respective control groups. In stage 1, both experimental groups were required to approach one of three platforms. The platforms were placed at the corners of a notional isosceles triangle in a circular pool. The correct platform was white, while the incorrect platforms were black and grey. In stage 2 , the platform array was placed into an equilateral triangular pool with one uniquely colored wall for Group Landmark Beacon and its control group and an isosceles pool for Group Shape Beacon groups. Schematic layouts of the pools and platforms in Experiment 3 are illustrated in Fig. 8. In the test trial the platforms were removed and participants were required to go to the area of the pool where the correct platform had been placed. From the results of Experiment 1 learning to approach a visually distinct platform might be expected to block learning about the position of the platform 
$\mathrm{N}$

Stage 1

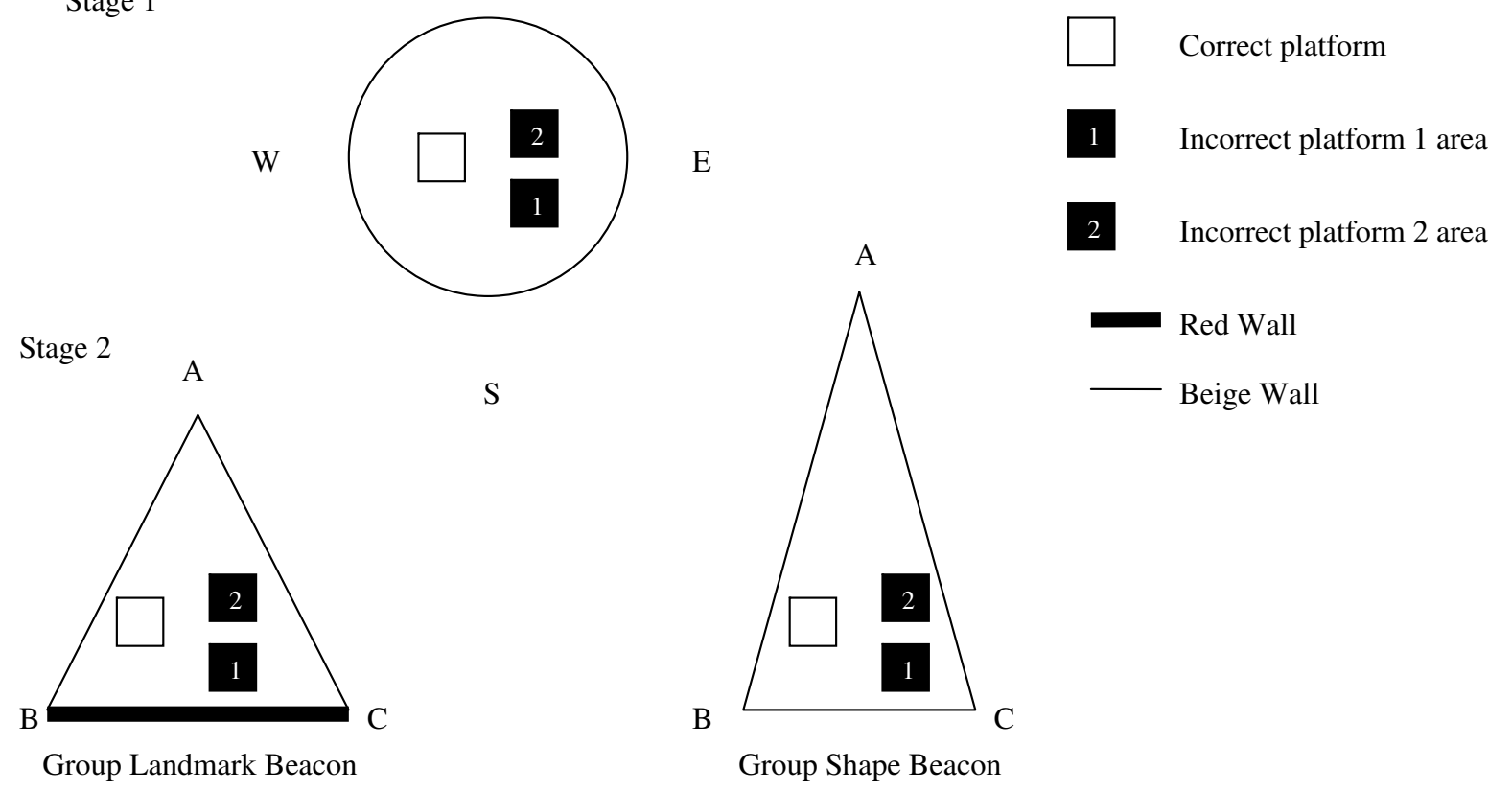

Fig. 8. Pool shape and platform position in stage 1 and in stage 2 for Group Landmark Beacon and Group Shape Beacon.

in terms of the red wall but not in terms of the shape of the isosceles triangle. In Experiment 2, learning about the geometric cue was blocked. It was suggested that the disruption of learning seen in Group Shape Shape was due to the pre-exposure to the shape of the platform array blocking learning about the shape of the pool.

A simpler explanation for the poor spatial learning displayed by Group Shape Shape in Experiment 2 might be that the position of the correct platform was much further from the pool corner, compared to the distance between the incorrect platform 1 and its nearest corner. If participants were using a strategy of searching close to the walls, then Group Shape Shape would have spent less time in the correct platform area compared to the incorrect platform area. If this argument is correct then Group Shape Beacon should again show no evidence of having learnt the relationship between the correct platform's position and the shape of the pool.

\section{Methods}

\section{Participants}

Participants were 54 undergraduate students, receiving payment of $£ 1.50$ for participation, divided equally into 4 groups: Group Landmark Beacon, Group Landmark Beacon Control, Group Shape Beacon and Group Shape Beacon Control. Within each experimental group, there were 4 males and 8 females; within the control groups there were 5 males and 10 females. The mean age was 25.6 years (range $18-$ 37 years). Participants were not permitted to take part in Experiment 3 if they had previously completed either Experiment 1 or 2.

\section{Materials and apparatus}

Materials and apparatus details were the same as in Experiment 2, with the exception that the correct platform was white and the incorrect platforms were both black and grey.

\section{Procedure}

All procedural details were the same as Experiment 2. 
Results and discussion

The Groups Landmark Beacon and Shape Beacon were analysed separately with their respective control groups to assess the effects of pre-exposing the participants to a visible platform.

\section{Group Landmark Beacon and Landmark Beacon Control}

The mean escape latencies for Group Landmark Beacon decreased over the course of stage 1 training (Trial $1 M=16.61, S D=7.90$, and Trial $12 M=7.16, S D=7.03$ ). A one-way repeated measures ANOVA was performed on the escape latencies of Group Landmark Beacon, with trial (within) the independent variable. There was a significant effect of trial, $F(11,121)=5.60$.

The mean escape latencies in stage 2 remained constant for Group Landmark Beacon (Trial 1 $M=8.53, S D=3.95$, and Trial $12 M=6.00, S D=2.37$ ) but decreased over trials for Group Landmark Beacon Control (Trial $1 M=14.55, S D=8.25$, and Trial $12 M=5.43, S D=2.05$ ). The group scores were analysed using a two-way mixed design ANOVA with group (between) and trial (within) the independent variables. There was no main effect of group, $F<1$, a significant effect of trial, $F(11,275)=10.89$, and a significant interaction between group and trial, $F(11,275)=4.02$. Further analysis of the simple main effects revealed that there was an effect of group only on trials $1, F(1,300)=22.32$, and 2 , $F(1,300)=7.51$, where escape latencies for Group Landmark Beacon Control exceeded those of Group Landmark Beacon. There was a significant effect of trial for Group Landmark Beacon Control, $F(11,275)=13.44$, but not for Group Landmark Beacon, $F(11,275)=1.47$.

The group means for the time spent in the correct and incorrect platform areas, represented as a percentage of the total time during the test trials, are shown in the left hand panel of Fig. 9. The percentage time spent in the correct platform was higher for both groups than in incorrect platform 2 but not incorrect platform 1. A two-way mixed design ANOVA with percentage time as the dependent variable and group (between) and platform type (within) as the independent variables confirmed this impression. There was a significant main effect of group, $F(1,25)=15.27$, a significant effect of platform type, $F(2,50)=3.41$, but a non-significant interaction between group and platform type, $F<1$. Newman-Keuls tests on the time spent in the platform areas by both groups combined confirmed that participants spent more time in the correct platform than in incorrect platform $2, q^{n k}(50)=3.57$, but not incorrect platform $1, q^{n k}(50)=0.89$.
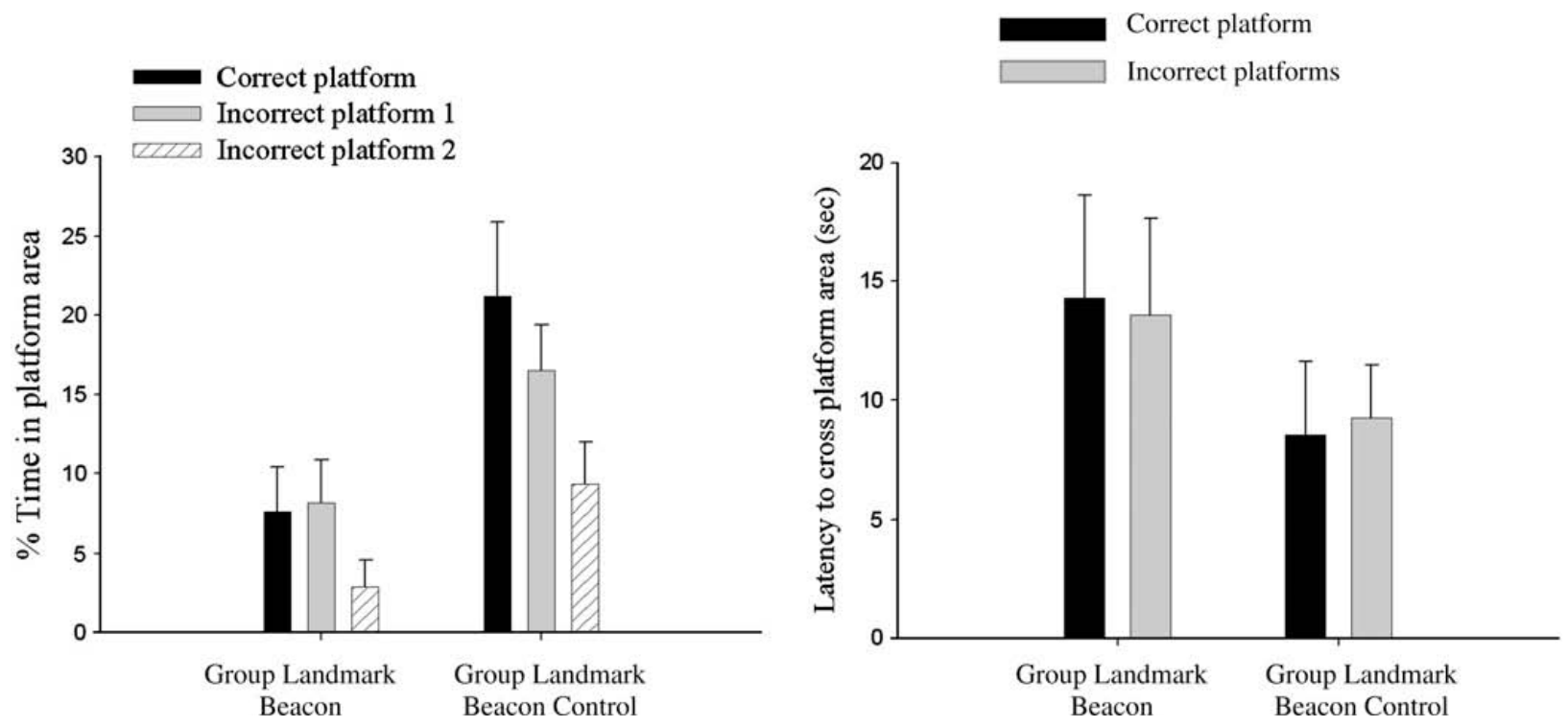

Fig. 9. Mean percentage time spent in correct (black bars) and incorrect (grey bars) platforms for Group Landmark Beacon and Group Landmark Beacon Control during test trial (left hand panel). Mean latency to cross correct (black bars) and incorrect (grey bars) platforms for Group Landmark Beacon and Group Landmark Beacon Control during test trial (right hand panel). The standard error bars are the standard error of the mean. 
The group mean for the latency to cross the correct and incorrect platforms during the test trial are shown in the right hand panel of Fig. 9. The latency to cross the correct platform was similar to the latency to cross the incorrect platforms for both Group Landmark Beacon and Group Landmark Beacon Control. A two-way mixed design ANOVA with latency to cross the platform as the dependent variable and group (between) and platform type (within) as the independent variables confirmed this impression. There was no main effect of group, $F(1,25)=1.71$, no effect of platform type, $F<1$, and no interaction between Group and Platform Type, $F<1$.

\section{Group Shape Beacon and Shape Beacon Control}

The mean escape latencies for Group Shape Beacon decreased over the course of stage 1 training (Trial $1 M=11.37, S D=3.06$, and Trial $12 M=4.26, S D=0.84$ ). A one way repeated measures ANOVA was performed on the escape latencies with trial (within) the independent variable. There was a significant effect of trial, $F(11,121)=5.28$.

The mean escape latencies in stage 2 stayed constant across trials for Group Shape Beacon (Trial 1 $M=8.09, S D=3.02$, and Trial $12 M=6.06, S D=2.11)$ but decreased over the trials for Group Shape Beacon Control (Trial $1 M=18.37, S D=14.91$, and Trial $12 M=5.71, S D=2.07$ ). The groups were compared using a two-way mixed design ANOVA with group (between) and trial (within) the independent variables. There was a significant main effect of group, $F(1,25)=6.36$, a significant effect of trial, $F(11,275)=2.83$, and a significant interaction between group and trial, $F(11,275)=1.99$. Further analysis of the simple main effects revealed that there was an effect of group only on trial 1 , $F(1,300)=10.88$, trial $2, F(1,300)=11.26$ and trial $5, F(1,300)=5.56$, where escape latencies for Group Shape Beacon Control exceeded the times of Group Shape Beacon. There was a significant effect of trial for Group Shape Beacon Control, $F(11,275)=4.73$, but not for Group Shape Beacon, $F<1$.

The group means for the time spent in the correct and incorrect platforms, represented as a percentage of the total time during the test trials, are shown in the left hand panel of Fig. 10. The percentage time spent was higher in the correct platform compared to the incorrect platforms for both Group Shape Beacon and Group Shape Beacon Control. A two-way mixed design ANOVA with percentage time as the dependent variable and group (between) and platform type (within) as the independent variables confirmed this appearance. There was no effect of group, $F(1,25)=2.61$, an effect of platform type, $F(2,50)=16.49$, and a non-significant interaction between group and platform type, $F<1$. Newman-Keuls tests confirmed that participants of both groups spent more time in the correct platform than in both incorrect platform $1, q^{n k}(50)=6.31$, and incorrect platform $2, q^{n k}(50)=7.65$.
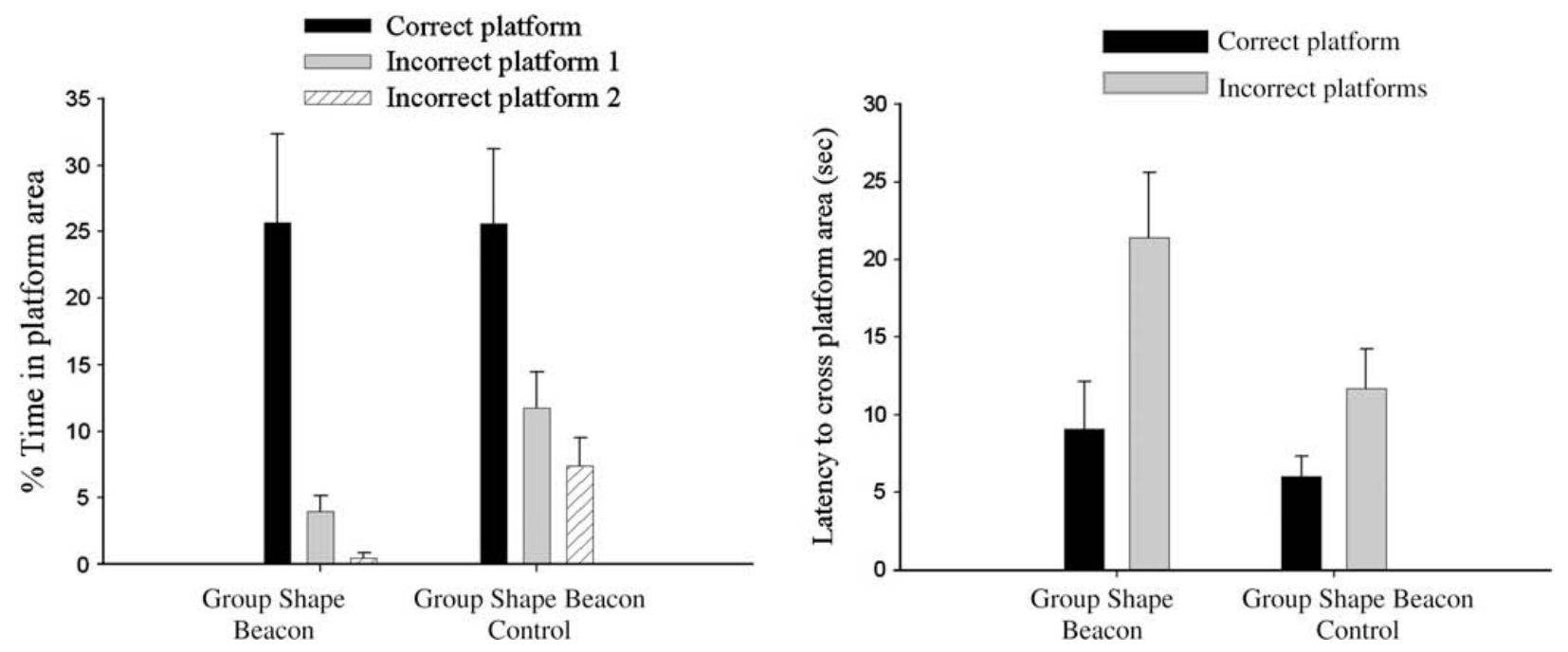

Fig. 10. Mean percentage time spent in correct (black bars) and incorrect (grey bars) platforms for Group Shape Beacon and Group Shape Beacon Control during test trial (left hand panel). Mean latency to cross correct (black bars) and incorrect (grey bars) platforms for Group Shape Beacon and Group Shape Beacon Control during test trial (right hand panel). The standard error bars are the standard error of the mean. 
The group mean for the latency to cross the correct and incorrect platforms during the test trial are shown in the right hand panel of Fig. 10. The latency to cross the correct platform was shorter than the latency to cross the incorrect platform for both Group Shape Beacon and Group Shape Beacon Control. A two-way mixed design ANOVA with latency to cross the platform as the dependent variable and group (between) and platform type (within) as the independent variables confirmed this impression. There was a significant main effect of group, $F(1,25)=7.78$, and platform type, $F(1,25)=7.70$, but the interaction between Group and Platform Type was not significant, $F(1,25)=1.06$.

The results of Experiment 3 were very similar to those of Experiment 1. The participants in both Group Shape Beacon and Group Shape Beacon Control demonstrated that they had learned the position of the correct platform in relation to the shape of the pool by spending significantly longer in the correct platform area, and the participants' latency to cross the correct platform was shorter than the latency to cross the incorrect platform areas. The presence of the visually distinct platform disrupted the ability of the participants of both Group Landmark Beacon and Group Landmark Beacon Control to learn the spatial relationship between the platform position and the red wall of the equilateral triangle, as neither measure of spatial learning demonstrated a preference for the correct platform. As with Experiment 1, it can not be specified that pre-exposure in stage 1 to the visible platform blocked spatial learning, as mere exposure to the visible platform in stage 2 resulted in equally poor spatial learning in the overshadowing control group. The results replicate findings by Redhead and Hamilton (2007) that a visible platform disrupts spatial learning based on non-geometric cues.

The results of Group Shape Beacon suggest that those of Group Shape Shape in Experiment 2 were not due to the positions of the platforms in relation to the corners of the pool or the restricted path taken by the participants. Given the two sets of results, it seems more likely that pre-exposure to the shape of the platform array blocked learning about the platform's position in relation to the shape of the platform. Overall the results suggest that although geometric cues are processed separately from non-geometric cues, they appear to compete with other geometric cues in a way described by associative models (e.g. Rescorla \& Wagner, 1972).

There were incidences of group differences between the respective experimental and control groups. Longer early latencies for the control groups in stage 2 can be explained by the control groups lack of previous training. By the end of the stage, there was no difference between the latencies of the experimental and control groups. During test trials, Group Landmark Beacon Control spent more time in the platform areas than Group Landmark Beacon. It is not possible to say why this might be the case, but crucially time in the correct platform was at least no different to incorrect platform 1 for both groups. Latency to cross either platform type was longer for Group Shape Beacon. Again it is not easy to explain this group difference, however, for both groups' participants crossed the correct platform area before the incorrect platform areas.

\section{General discussion}

The results suggest that learning about the spatial relationship between the position of the platform and both geometric and non-geometric landmarks can be disrupted within a blocking design. In Experiments 1 and 3, exposure to a visually distinct platform disrupted learning about the spatial relationship between the position of the platform and the red wall of the pool. These findings are consistent with both animal (e.g., Redhead et al., 1997) and human findings (e.g., Chamizo et al., 2003; Redhead \& Hamilton, 2007). It is not possible to state that this disruption was due to the pre-exposure in stage 1 , as the respective control groups also showed disrupted spatial learning. Group Landmark Control in Experiment 1 did show faster latency to cross the correct platform area but did not spend any more time than in the incorrect area. It could be argued that participants are simply unable to learn the position of a platform in relation to the non-geometric cue of a red wall. However, the performance of Groups Landmark Shape and Landmark Shape Control in Experiment 2, where good spatial learning based on the non-geometric cues was demonstrated, shows this not to be the case. We are left to conclude that the presence of a visible platform disrupts learning about the non-geometric cue in both a blocking and overshadowing design. 
The results of Group Shape Shape in Experiment 2 demonstrate that learning about the shape of a pool can be blocked by pre-exposure to a competing geometric cue created by the spatial configuration of an array of identical beacons. On this occasion, the mere presence of the array in stage 2 was not sufficient to disrupt learning of the shape of the pool by Group Shape Shape Control. There have been other demonstrations of geometry being disrupted by non-geometric cues (e.g., Gray, Bloomfield, Ferrey, Spetch, \& Sturdy, 2005; Pearce et al., 2006), but this is the first time pre-exposure to a geometric cue has been shown to block learning about another separate geometric cue.

Our findings are in contrast to Doeller and Burgess (2008) who found no blocking between geometric cues. They presented two differently coloured halves of a single circular boundary to participants in a computer generated environment. In the initial stage, each half of the boundary formed a stable spatial relationship with its own distinct set of goals while maintaining an unstable spatial relationship with the alternative half's cues. In the compound stage, both halves formed a stable spatial relationship with all cues. During the test stage, only one half of the boundary was presented at a time, and participants were required to locate both the cues which had a stable spatial relationship with that boundary half throughout and the cues for which this was true only during compound training. Participants were equally able to locate both sets of cues.

Although these results are at odds with our own, there are two major differences in the procedure of the studies. Firstly, the Doeller and Burgess (2008) design is more akin to a learned irrelevance paradigm than to a blocking design, as the blocked geometric cue was present but irrelevant to the position of the cues in stage 1. It could be argued that the boundary wall was also present in our studies during stage 1, but, because it was circular it was deemed irrelevant to the position of the platforms. Even if this was the case, it would seem blocking is the more important factor regarding poor spatial learning in Group Shape Shape given the results of Group Shape in Experiment 1. Here training with a circular maze did not disrupt subsequent use of the geometric cues. Whether the impairment in spatial learning was due to blocking, learned irrelevance or a combination, it was observed in Group Shape Shape but not the corresponding group in Doeller and Burgess's study. The second difference between the studies might thus be the key to the contrasting results. In the current study, the geometric cues were separate, the shape of the platform array and the shape of the pool, but for Doeller and Burgess the geometric cues were two halves of the same boundary. In order to see blocking, it may be necessary to have separate geometric cues. Further research would be necessary to answer this question.

The results of Experiments 1, 2 and 3 further suggest that there is no interaction between geometric and non-geometric cues. The pre-exposed non-geometric cue of the visually distinct platform did not disrupt subsequent learning about the shape of pool in Experiment 1 and 3. The pre-exposed geometric cue of the platform array in Experiment 2 did not disrupt learning about the position of the platform in terms of the non-geometric red wall. Similar lack of interaction between geometric and non-geometric cues has been found (e.g., Doeller \& Burgess, 2008; Redhead \& Hamilton, 2007) and taken as evidence that geometric cues are impervious to disruption by other types of cues (e.g., Cheng, 1986; Gallistel, 1990; Margules \& Gallistel, 1988; Wang \& Spelke, 2002).

Recent evidence from animal work (e.g., Gray et al., 2006; Pearce et al., 2006), has suggested that under certain conditions geometric and non-geometric cues can interact. Pearce et al. suggested that these interactions vary according to the salience of the geometric cues. Learning about the geometric cues formed by a kite shaped pool was potentiated by the presence of a black wall while those formed by a rectangular shaped pool were overshadowed. In the current paper, although there is no direct evidence for interference between geometric and non-geometric cues, some of the findings from Experiment 3 pose a problem for the notion that geometric and non-geometric cues are processed separately. During stage 1, even though the correct platform was visually distinct from the other two platforms, it could also be identified by its position within the array. If non-geometric and geometric cues are processed separately, then it would be expected that both types of cue would have been employed to identify the correct platform. Thus it might be expected that both Group Landmark Beacon and Group Shape Beacon would have been unable to identify the position of the correct platform. The visually distinct platform might have been expected to have disrupted learning about the red wall and the position of the platform within the platform array to have disrupted learning about the shape of the pool. Group Shape Beacon, however, demonstrated that they had learned the position 
of the platform in terms of the shape of the pool by spending more time in the correct platform than in either of the incorrect platforms. Gibson, Wilks, and Kelly (2007) demonstrated that rats can be trained to locate a visually distinct object within a rectangular array of objects, but, when the objects are replaced by four identical objects, the rats do not spend more time searching the objects in the correct position.

The results of Experiment 3 can be taken as similar evidence that the non-geometric cue of the uniquely colored platform overshadowed the geometric cue of the position of the platform within the array. Additional test trials, at the end of stage 1, using either 3 identical platforms or platforms placed in an equilateral array would be required to ascertain the nature of the interaction between these geometric and non-geometric cues. Such additional probe trials would have been disruptive to blocking as they would have reduced the associative strength of the stage 1 stimuli and thus were not run.

Assuming geometric and non-geometric cues do interact, it is worth discussing whether associative processes could describe the results observed. Miller and Shettleworth (2007) suggested an associative model with which they have been able to explain the absence and presence of blocking seen between geometric and non-geometric cues (e.g., Pearce et al., 2001; Pearce et al., 2006). The authors posit a concept they term Featural Enhancement whereby the presence of a visible platform will increase the probability of choosing one corner over another. The associative strength of the geometry of the correct corner would increase faster than it would normally occur without the beacon due to the corner's higher contingency with reward. On testing in the absence of the beacon, there is a reduction of blocking as seen in Groups Shape and Shape Control of Experiment 1.

Conversely, when a highly salient feature is present in the incorrect corner, such as dark walls in a rectangular watermaze used by Pearce et al. (2006), increasing searching in the incorrect corner would hasten the development of inhibition to the geometric cues, resulting in blocking. In terms of this second prediction, it might be argued that the presence of two possible correct platforms at the base of the isosceles shaped array was more attractive than one at the apex, and thus inhibition to the geometric cues at the incorrect corner was increased and blocking was observed in Group Shape Shape. However, Miller and Shettleworth (2007) emphasise the difference between geometrically ambiguous and unambiguous enclosures. Blocking was observed in Pearce et al. (2006), where the enclosure was rectangular, and two corners had similar geometric cues. Inhibition to the incorrect corner would be expected by the model to generalise to the geometrically equivalent incorrect corner, resulting in blocking. In the isosceles triangle of Group Shape Shape, which was used because it had no geometrically ambiguous corners, any inhibition of geometric cues at the incorrect corner should help to disambiguate the correct corner and so improve learning rather than blocking it. Thus it is difficult to see how Miller and Shettleworth's model could explain the findings from Group Shape Shape. It would also have difficulty explaining the differential blocking seen in the Landmark and Shape groups without assuming featural enhancement is different for geometric and non-geometric cues.

Prior research has shown that more salient cues are less likely to become blocked by less salient pre-exposed cues (e. g., Dente \& Krusche, 2006; Hall, Mackintosh, Goodall, \& dal Martello, 1977). In order for salience of the to-be-blocked cue to explain the results of Experiment 1, we would have to conclude that the visually distinct platform was less salient than the shape of the isosceles pool but more salient than the red wall of the equilateral pool. To explain the results of Experiment 2, the spatial array of platforms would have to be more salient than the shape of the isosceles pool and less salient than the red wall in the equilateral pool. Given the results of Experiment 1, this would not be possible.

Without some restrictions on the interaction between geometric and non-geometric cues, it would seem the results can not all be explained via associative processes. It may, therefore, be necessary to explore non-associative mechanisms to explain the apparent blocking of geometric cues seen in Experiment 2. The array of identical platforms used in Experiment 2 might be described as being a nested environment within the arena. Wang and Brockmole (2003) found that human participants navigating between nested environments seemed to switch attention between environments, only updating the approaching environment and losing track of old environments. The authors suggest that spatial updating in nested environments does not occur for all environments at the same time. Updating their position within the platform array might have meant participants in Group Shape Shape were unable to update their position within the pool as a whole. If this was the case, why would the array 
not also block learning about the red wall in Group Landmark Shape? Once on the platform it would have been far easier to update their position within the pool using the relative positions of colored walls from a static point compared to the shape of the pool, as illustrated by Fig. 2. Thus, while Group Landmark Shape might have been able to learn an additional navigation strategy waiting on the platform, Group Shape Shape was less likely to have been able to have done so. Further experiments providing eye tracking information would test the validity of such an explanation.

The results of Experiments 1 demonstrated that pre-exposure to non-geometric cues will block learning about other non-geometric cues within both a blocking and overshadowing design but do not disrupt geometric cues. These results could be explained if geometric and non-geometric cues were processed separately. The results could also be explained by associative processes if the shape of the isosceles platform was considered to be more salient than the other cues and thus less likely to be blocked. Experiment 2 provided novel evidence that geometric cues will disrupt learning about other geometric cues but not non-geometric cues and again supports the notion that geometric and non-geometric cues are processed separately. Without the notion of separate processing, the results do not fit with associative predictions but could be explained via alternative attentional processes. The results of Experiment 3 repeat the findings of Experiment 1 but also suggest that the non-geometric cue of a visually distinctive platform may have overshadowed the less salient geometric cue of the platform's position within the platform array.

The results offer novel evidence that geometric cues can be blocked by pre-exposed alternative geometric cues. However, taken as a whole, the results can not be explained by an associative description of blocking. The majority of the results suggest that there is no interaction between geometric and non-geometric cues, but the findings of Experiment 3 suggest that salient non-geometric cues can disrupt learning of less salient geometric cues. Thus any proposed modular system must allow for interaction between the geometric and non-geometric cues.

\section{References}

Benhamou, S., \& Poucet, B. (1998). Landmark use by navigating rats (Rattus norvegicus): Contrasting geometric and featural information. Journal of Comparative Psychology, 112, 317-322.

Chamizo, V. D., Aznar-Casanova, J. A., \& Artigas, A. A. (2003). Human overshadowing in a virtual pool: Simple guidance is a good competitor against locale learning. Learning and Motivation, 34, 262-281.

Chamizo, V. D. (2003). Acquisition of knowledge about spatial location: Assessing the generality of the mechanism of learning. Quarterly Journal of Experimental Psychology, 56B, 102-113.

Cheng, K. (1986). A purely geometric module in the rat's spatial representation. Cognition, 23, 149-178.

Cheng, K. (2005). Reflections on geometry and navigation. Connection Science, 17, 5-21.

Dente, S. E., \& Krusche, J. K. (2006). Attention and salience in associative blocking. Learning E Behaviour, 34, 285-304.

Doeller, C. F., \& Burgess, N. (2008). Distinct error-correcting and incidental learning of location relative to landmarks and boundaries. Proceedings of the National Academy of Sciences of the USA, 105, 5909-5914.

Doeller, C. F., King, J. A., \& Burgess, N. (2008). Parallel striatal and hippocampal systems for landmarks and boundaries in spatial memory. Proceedings of the National Academy of Sciences of the USA, 105, 5915-5920.

Gallistel, C. R. (1990). The organisation of learning. Cambridge, MA: MIT Press.

Gibson, B. M., Wilks, T. J., \& Kelly, D. M. (2007). Rats (Rattus norvegicus) encode the shape of an array of discrete objects. Journal of Comparative Psychology, 121, 130-144.

Gray, E. R., Bloomfield, L. L., Ferrey, A., Spetch, M. L., \& Sturdy, C. B. (2005). Spatial encoding in mountain chickadees: Features overshadow geometry. Biology Letters, 1, 314-317.

Hall, G., Mackintosh, N. J., Goodall, G., \& dal Martello, M. (1977). Loss of control by a less valid or by a less salient stimulus compounded with a better predictor of reinforcement. Learning $\mathcal{E}$ Motivation, 8, 145-158.

Hamilton, D. A., \& Sutherland, R. J. (1999). Blocking in human place learning: Evidence from virtual navigation. Psychobiology, 27, 453-461.

Hayward, A., Good, M. A., \& Pearce, J. M. (2004). Failure of a landmark to restrict spatial learning based on the shape of the environment. Quarterly Journal of Experimental Psychology, 57B, 289-314.

Hayward, A., McGregor, A., Good, M. A., \& Pearce, J. M. (2003). Absence of overshadowing and blocking between landmarks and geometric cues provided by the shape of a test arena. Quarterly Journal of Experimental Psychology, 56B, 114-126.

Keppel, G. (1973). Design and analysis: A researchers' handbook. Englewood Cliffs, NJ: Prentice-Hall.

Margules, J., \& Gallistel, R. (1988). Heading in the rat: Determination by environmental shape. Animal Learning and Behaviour, 16 , 404-410.

McGregor, A., Hayward, A. J., Pearce, J. M., \& Good, M. A. (2004). Hippocampal lesions disrupt navigation based on the shape of the environment. Journal of Experimental Psychology: Animal Behavior Processes, 118, 1011-1021.

Miller, N. Y., \& Shettleworth, S. J. (2007). Learning about environmental geometry: An associative model. Journal of Experimental Psychology: Animal Behavior Processes, 33, 191-212.

Pearce, J. M., Graham, M., Good, M. A., Jones, P. M., \& McGregor, A. (2006). Potentiation, overshadowing and blocking of spatial learning based on the shape of the environment. Journal of Experimental Psychology: Animal Behavior Processes, 32, $201-214$. 
Pearce, J. M., Ward-Robinson, J., Aydin, A., Good, M., \& Fussell, C. (2001). Influence of a beacon on spatial learning based on the shape of the test environment. Journal of Experimental Psychology: Animal Behavior Processes, 27, 329-344.

Redhead, E. S., \& Hamilton, D. (2007). Interaction between locale and taxon strategies in human spatial learning. Learning and Motivation, 38, 262-283.

Redhead, E. S., Roberts, A., Good, M., \& Pearce, J. M. (1997). Interaction between piloting and beacon homing in a swimming pool. Journal of Experimental Psychology: Animal Behavior Processes, 23(3), 240-250.

Rescorla, R. A., \& Wagner, A. R. (1972). A theory of Pavlovian conditioning: Variations in the effectiveness of reinforcement and non-reinforcement. In A. H. Black \& W. F. Prokasy (Eds.), Classical conditioning II: Current research and theory (pp. 64-99). New York: Appleton-Century-Crofts.

Roberts, A. D. L., \& Pearce, J. M. (1999). Blocking in the Morris swimming pool. Journal of Experimental Psychology: Animal Behavior Processes, 25, 225-235.

Spetch, M. L., Cheng, K., \& MacDonald, S. E. (1996). Learning the configuration of a landmark array. Part I: Touch-screen studies with pigeons and humans. Journal of Comparative Psychology, 110, 55-68.

Wang, R. F., \& Spelke, E. S. (2000). Updating egocentric representations in human navigation. Cognition, 77, 215-250.

Wang, R. F., \& Spelke, E. S. (2002). Human spatial representation: Insights from animals. Trends in Cognitive Science, 6, 376-382.

Wang, R. F., \& Brockmole, J. R. (2003). Human navigation in nested environments. Journal of Experimental Psychology: Learning Memory and Cognition, 29, 398-404. 